\section{P74 THE IMPACT OF RECONFIGURING ACUTE HOSPITAL SERVICES ON HOSPITAL-TREATED SELF-HARM: A BEFORE-AND-AFTER STUDY}

${ }^{1} E$ Griffin, ${ }^{1} \mathrm{C}$ Murphy, ${ }^{2} \mathrm{IJ}$ Perry, ${ }^{2} \mathrm{~B}$ Lynch*, ${ }^{1,2} \mathrm{E}$ Arensman, ${ }^{2,3} \mathrm{P}$ Corcoran. ${ }^{1}$ National SelfHarm Registry Ireland, National Suicide Research Foundation, Cork, Ireland; ${ }^{2}$ Department of Epidemiology and Public Health, University College Cork, Cork, Ireland; ${ }^{3}$ Department of Obstetrics and Gynaecology, University College Cork, Cork, Ireland

\subsection{6/jech-2017-SSMAbstracts. 175}

Background Health services in Ireland and the United Kingdom have developed strategies to reduce the number of acute hospitals. This has involved the centralisation of services to centres of excellence along with the reconfiguration of smaller hospitals to urgent care centres - with reduced emergency department (ED) hours. However, the evidence base for improved patient outcomes is limited. We aimed to assess the impact of the reconfiguration of a hospital group in Ireland in terms of the burden of hospital-treated self-harm on each hospital and the clinical management of individual self-harm patients.

Methods The study was conducted in three Mid-Western regional hospitals in Ireland. The reconfiguration in April 2009 involved two hospitals (B and C) reducing the operation of their EDs while services at a third hospital (A) remained unchanged. As part of the National Self-Harm Registry Ireland, data were recorded relating to all self-harm presentations during the period January 2004 to April 2014. We used Poisson regression analysis to assess changes in the hospital burden and clinical management of self-harm.

Results During the study period there were 9223 self-harm presentations to the EDs of the three hospitals. Hospital A received the majority (75\%), with Hospitals $\mathrm{B}$ and $\mathrm{C}$ receiving $14 \%$ and $11 \%$ of presentations, respectively. The reconfiguration was associated with a marked increase in the rate of selfharm presentations at Hospital A, from a rate of 46.0 to 65.2 per month $[+19.2$ (95\% CI 16.2, 22.4)]. This increase was approximately equivalent to the decreases at Hospitals B $[-7.7(-8.4,-6.8)]$ and C $[-9.4(-9.9,-8.4)]$. Despite this large increase in presentations, there was only a small increase in admissions into Hospital A [+3.4 per month $(1.4,5.5)]$.

Conclusion The cumulative decrease in self-harm presentations at the hospitals reconfigured to have reduced ED services was of similar magnitude to the increase at the other hospital's ED. This suggests that such reconfiguration does not reduce self-harm presentations to hospital EDs but shifts the burden of cases to other hospitals. The disproportionately small impact on hospital admissions suggests that the management of self-harm patients may change in response to increased numbers presenting.

This is the first known study to quantify the impact of acute hospital reconfiguration on patterns of self-harm attendances, highlighting changes in the management of self-harm specifically. Patient outcomes following hospital reconfiguration should be an ongoing research priority.

\section{P75 A COST-EFFECTIVENESS ANALYSIS OF IMPROVING ACCESS TO PSYCHOLOGICAL THERAPIES IN BRITISH SIGN LANGUAGE (BSL)}

${ }^{1} \mathrm{GE}$ Shields ${ }^{*}{ }^{2} \mathrm{KD}$ Rogers, ${ }^{2} \mathrm{~A}$ Young, ${ }^{1} \mathrm{D}$ Buck, ${ }^{1} \mathrm{LM}$ Davies. ${ }^{1}$ Centre for Health Economics, University of Manchester and MAHSC, Manchester, UK; ${ }^{2}$ Division of Nursing, Midwifery and Social Work, University of Manchester and MAHSC, Manchester, UK

\subsection{6/jech-2017-SSMAbstracts. 176}

Background Deaf BSL users can access standard Improving Access to Psychological Therapies (standard-IAPT) services with/without interpreter assistance in the UK. Some areas use specialist BSL-IAPT services, delivered by qualified IAPT workers who are fluent in BSL, addressing cultural and linguistic needs of Deaf users. This NIHR HS and DR funded study (Ref: 12/136/79) assessed the cost-effectiveness of BSL-IAPT, versus standard-IAPT services, for Deaf BSL users.

Methods A combined decision tree (initial IAPT intervention period) and Markov model (longer-term costs and health benefits) used a NHS and social care perspective to estimate the incremental cost/quality-adjusted life-year (QALY) gained and probability that BSL-IAPT is cost-effective. The probability of cost-effectiveness was estimated for willingness to pay thresholds from $£ 0$ to $£ 30000$ to gain one QALY. The model structure was developed from published literature and extensive discussion with the research team and advisory group. Both included Deaf people. Data sources for clinical effectiveness, direct costs and utilities included systematic reviews, IAPT databases and new studies with Deaf BSL users. The timeframe was 5 years with costs and QALYs discounted. Probabilistic and deterministic sensitivity analyses assessed parameter and structural uncertainty.

Results BSL-IAPT is associated with a net saving of $£ 240$ (95 th percentiles $£ 2,303, £ 935$ ) and little difference in QALYs (net QALY 0.001; 95th percentiles -0.009, 0.013). In both cases the 95th percentiles cross zero, indicating uncertainty about the results. However, cost-effectiveness acceptability analysis indicated probability above $50 \%$ that BSL-IAPT is cost effective, even if decision makers are not prepared to pay to gain one QALY. Most sensitivity analyses also indicated that BSL-IAPT was likely to be cost effective. Exceptions were if it was assumed that (i) there was no additional cost for interpreters in Standard-IAPT cost; (ii) BSL-IAPT provides more high intensity care than currently, though the latter is very uncertain due to limited data.

Conclusion The primary analysis indicated that BSL-IAPT may be more cost effective than Standard IAPT. Limited data and reliance on a small sample of IAPT data means that the robustness of the results are uncertain. Nevertheless, this is the first economic evaluation of a healthcare for depression and anxiety interventions for Deaf BSL users. It provides initial information to support healthcare commissioners achieve increased access to effective and efficient psychological therapies for depression in Deaf people. The study also highlights the need for new research to prospectively collect robust data. 